\title{
Extractos vegetales: una alternativa para el control de enfermedades en el cultivo de cacao (Theobroma cacao)
}

\author{
Plant extracts: an alternative for the control of diseases in cacao (Theobroma cacao)
}

Raquel Guerrero ${ }^{*}$ * ID

Universidad Técnica Estatal de Quevedo

Gabriela Risco ${ }^{2}$ iD

Universidad Técnica Estatal de Quevedo

Orly Cevallos 3

Universidad Técnica Estatal de Quevedo

Ronald Villamar 4 iD

Universidad Técnica Estatal de Quevedo

Sofía Peñaherrera ${ }^{5}$ iD

Universidad Técnica Estatal de Quevedo

Fecha recepción: 15 de junio de 2020

Fecha aceptación: 16 de julio de 2020

(1) 2020 Universidad de Cordoba. Este es un artículo de acceso abierto distribuido bajo los términos de la licencia Creative Commons Attribution License, que permite el uso ilimitado, distribución y reproducción en cualquier medio, siempre que el autor original y la fuente se acreditan.

\footnotetext{
1 Magister en Protección Vegetal, Universidad Técnica Estatal de Quevedo, Docente de la Facultad de Ciencias Pecuarias, Quevedo, Ecuador, rguerrero@uteq.edu.ec, ORCID0000-0002-7609-3480

2 Ingeniera Agropecuaria, Consultora RS, Quevedo, Ecuador, gabrielariscoagrosolutions@gmail.com, ORCID 0000-0002-3630-1329

3 Doctor en Recursos Naturales y Gestión Sostenible, Universidad Técnica Estatal de Quevedo, Docente de la Facultad de Ciencias Pecuarias, Quevedo, Ecuador fcevallos@uteq.edu.ec, ORCID0000-0002-4137-7133

${ }^{4}$ Doctor en Biología, Diversidad y Mejoramiento Integral de Plantas, Universidad Técnica Estatal de Quevedo, Docente de la Facultad de Ciencias Pecuarias, Quevedo, Ecuador rvillamart@uteq.edu.ec, ORCID0000-0003-

5 Magister en Agronomía, Instituto Nacional de Investigaciones Agropecuarias, Investigadora Estación Tropical Experimental Pichilingue, Quevedo, Ecuador loren80@gmail.com, ORCID 0000-0001-5055-4798
} 


\section{RESUMEN}

El cacao (Theobroma cacao) enfrenta durante cada uno de sus ciclos productivos patógenos que disminuyen considerablemente a su producción. Cada año fitopatógenos como Moniliophthora roreri y Phytophthora palmivora afectan directamente a las mazorcas y pueden llegar a generar graves pérdidas. Esta investigación se desarrolló con la finalidad de identificar especies vegetales locales cuyos extractos contengan compuestos que tienen la capacidad de reducir la germinación de las esporas de los patógenos, así como también el desarrollo de micelio de los mismos. Extractos de las especies Bledo (Amaranthus bledo), Coquito (Cyperus odoratus), Lechosa (Euphorbia hirta), Rosa de muerto (Tagetes minuta), Teatina (Scoparia dulcis) y Verdolaga (Portulaca oleracea) se utilizaron para enmendar medios de cultivos en concentraciones de 10, 20 y 30\%, sobre los cuales se inocularon suspensiones de esporas y discos de micelio de los patógenos. Transcurridos los periodos de incubación se evaluaron los porcentajes de germinación, porcentaje de inhibición de desarrollo del micelio, actividad fungicida de los extractos y su efecto sobre las estructuras de las hifas de los patógenos. Los resultados mostraron que todos los extractos en alguna medida influyeron en alguna medida en el desarrollo de los patógenos en todas las variables estudiadas, sin embargo, Amaranthus bledo y Euphorbia hirta fueron aquellas especies cuyo efecto fue notablemente superior a las otras especies en estudio, lo que indicaría que son

\section{ABSTRACT}

Cocoa (Theobroma cacao) faces pathogens during each of its productive cycles that considerably decrease its production. Every year plant pathogens like Moniliophthora roreri and Phytophthora palmivora directly affect the pods and can lead to serious losses. This research was developed in order to identify local plant species whose extracts contain compounds that have the ability to reduce the germination of pathogens spores, as well as the development of their mycelium. Extracts of the species amaranthus (Amaranthus bledo), rusty flat sedge (Cyperus odoratus), Lechosa (Euphorbia hirta), marigold (Tagetes minuta), sweet-broom (Scoparia dulcis) and purslane (Portulaca oleracea) were used to modificate culture media in concentrations of 10,20 and $30 \%$, on which spore suspensions and mycelium discs of the pathogens were inoculated. After the incubation periods, the percentages of germination, percentage of inhibition of mycelial development, fungicidal activity of the extracts and their effect on the structures of the hyphae of the pathogens were evaluated. The results showed that all the extracts to some extent influenced to some extent the development of the pathogens in all the variables studied, however, Amaranthus bledo and Euphorbia hirta were those species whose effect was notably superior to the other species under study, which would indicate that they are species with the potential to control pathogens at the field level. 
especies con potencial para controlar los patógenos a nivel de campo.

PALABRAS CLAVE: extractos, vegetales, control, enfermedades, cacao
KEYWORDS: extracts, botanicals, control, diseases, cocoa

\section{INTRODUCCIÓN}

El cacao (Theobroma cacao) es uno de los cutivos de mayor importancia económica para el Ecuador. La producción y rentabilidad del cacao y del banano están continuamente amenazadas por la presencia de patógenos de origen fúngico que disminuyen las cosechas. Dentro de estos patógenos se destacan los hongos: Moniliopththora roreri, causante de la moniliasis del cacao; y, Phytophthora palmivora, que provoca la pudrición negra de las mazorcas de cacao.

Debido al impacto de estos problemas fitopatológicos, es importante encontrar herramientas efectivas para su manejo. Las opciones de control disponibles se concentran principalmente en el constante uso de pesticidas de origen químico, que han provocado que los patógenos desarrollen resistencia, creando a su vez la necesidad de utilizar moléculas cada vez más tóxicas (Bateman, 2009). Adicionalmente, tanto los consumidores locales como los procedentes de mercados internacionales están cada vez más preocupados por la toxicidad y la cantidad de plaguicidas que se utilizan en los sistemas agrícolas lo que hace imperativo investigar alternativas para el control de las enfermedades que sean efectivas, y al mismo tiempo, menos contaminantes.

Una de estas alternativas es el uso de extractos de plantas que han presentado resultados prometedores. Los extractos vegetales tienen las ventajas de poseer un origen biológico, ser degradables y manifestar un mínimo impacto negativo sobre la salud humana y el medio ambiente (Isman, 2000). A nivel mundial muchos investigadores han demostrado los efectos negativos que tienen los extractos vegetales de ciertas especies sobre el desarrollo de fitopatógenos (Balandrin et al., 1985; Isman, 2006; Sporleder \& Lacey, 2013; Walia et al., 2014; Ten Hopen \& Krauss, 2016). El empleo de plantas que son consideradas malezas resultan una fuente económica y muy importante de obtención de extractos vegetales con actividad antimicrobiana (Zhou \& Yu, 2006). En nuestro país la información referente a esta temática es limitada, especialmente, aquella que relaciona los extractos vegetales con el control de los microorganismos fitopatógenos.

A través de esta investigación se buscó seleccionar plantas locales, cuyos extractos posean compuestos que tengan la capacidad de controlar el desarrollo in vitro de patógenos de importancia económica para el país, para ellos se llevaron a cabo ensayos a nivel in vitro para evaluar el efecto de los extractos sobre la germinación de las esporas de los patógenos, crecimiento, viabilidad y estructura del micelio de los fitopatógenos en medios de cultivo enmendados con extractos vegetales. 


\section{METODOLOGÍA}

Los ensayos y la colecta de plantas se realizaron en el Campus "La María" de la Universidad Técnica Estatal de Quevedo. Los ensayos se ejecutaron en el área de Microbiología del Laboratorio de Rumiología.

Cultivo de los patógenos

A partir de las colonias madre de M. roreri y P. palmivora que son parte del banco de cultivos de fitopatógenos de la UTEQ, se obtuvieron subcultivos en cajas de Petri contiendo medio PDA mediante repiques. Los cultivos se incubaron a $25^{\circ} \mathrm{C}+2^{\circ} \mathrm{C}$ durante ocho días para $\mathrm{P}$. palmivora y quince días para M. roreri.

Obtención de los extractos vegetales

La selección de plantas se realizó mediante el análisis de información bibiográfica para identificar plantas con potencial actividad antimicrobiana y/o antioxidante. Se seleccionaron seis plantas considerando la cantidad de compuestos con acción sobre fitopatógenos reportados en la literatura científica y que además sean parte de la flora de la región.

De cada planta se colectaron muestras de hojas, tallos y flores que fueron secadas en estufa a $35{ }^{\circ} \mathrm{C}$ hasta que alcanzaron peso constante. Las muestras secas se trituraron con un molino y se colocaron en una solución hidroetanólica (50\% agua destilada estéril y $50 \%$ etanol) a razón de $140 \mathrm{~g}$ de tejido vegetal triturado por litro de solución. Después de un periodo de ocho días se obtuvieron los extractos mediante filtración con papel filtro estéril, las soluciones obtenidas fueron sometidas a un proceso de eliminación de alcohol usando un rotoevaporador). Los extractos fueron conservados en botellas de vidrio estéril a $14^{\circ} \mathrm{C}$.

Evaluación del efecto de los extractos vegetales sobre la germinación de esporas

Para la obtención de las esporas del patógeno M. roreri se realizó subcultivos en placas de Petri conteniendo medio papa dextrosa agar (PDA) y agar jugo V8 (AV8) para el caso de P. palmivora. La incubación se realizó durante 10 días para P. palmivora y de 24 días para $\mathrm{M}$. roreri, ambas a $25^{\circ} \mathrm{C} \pm 2{ }^{\circ} \mathrm{C}$. Una vez confirmada para presencia de esporas y zoosporas (P. palmivora) estas se removieron con una asa de Drigalsky y se suspendieron en $20 \mathrm{ml}$ de agua destilada estéril con Tween 20 al $0.01 \%$. La suspensión se ajustó a una concentración de 1 x 106 esporas ml-1.

Los extractos fueron utilizados para enmendar los medios PDA (M. roreri) y AV8 (P. palmivora) diluidos y a una temperatura de $55{ }^{\circ} \mathrm{C}$, se prepararon de cada uno tres concentraciones: 10,20 y $30 \%$ v/v. Adicionalmente, se preparó medio de cultivo sin enmendar para el control.

La inoculación de M. roreri y P. palmivora, se realizó distribuyendo $100 \mu \mathrm{L}$ de la suspensión de esporas sobre la superficie del medio de cultivo. Por cada extracto/concentración se inocularon diez placas de Petri; además, se inoculó el patógeno en diez placas con medio PDA y AV8 (control). La incubación se realizó a una temperatura de $25{ }^{\circ} \mathrm{C}+2{ }^{\circ} \mathrm{C}$. Las evaluaciones se realizaron a las 24 horas después de la inoculación para $\mathrm{M}$. roreri; y, a las cinco horas después de la inoculación para P. palmivora.

Para ello, en cada ocasión se realizaron observaciones al microscopio (lente objetivo 40 X), y cuando el control alcanzó el $100 \%$ de germinación se detuvo la germinación en todos los tratamientos colocando cuatro gotas de una solución de Trypan Blue al $0.4 \%$, se diez campos ópticos por cada tratamiento para realizar contaje de cien esporas (germinadas y no germinadas) un campo por cada caja de Petri y con estos datos se calcularon los porcentajes de germinación. Siendo por tanto de cada cien esporas observadas:

$\mathrm{N}^{\circ}$ esporas germinadas $=\%$ esporas germinadas

$\mathrm{N}^{\circ}$ esporas no germinadas $=\%$ esporas no germinadas

Evaluación del efecto de extractos vegetales sobre el desarrollo micelial de los patógenos

Se inocularon los patógenos en los medio 
de cultivo PDA (M. roreri) y AV8 (P. palmivora) enmendados previamente con los extractos vegetales en tres concentraciones diferentes: 10, 20 y $30 \%$ v/v. Inicialmente, se obtuvieron cultivos de los patógenos en PDA y en AV8 (sólo para P. palmivora). Se inocularon diez placas de Petri por cada uno de los extractos/concentración. La inoculación se realizó colocando discos de micelio de los patógenos (de $5 \mathrm{~mm}$ de diámetro) en el centro de cada placa de Petri previamente envasada con $20 \mathrm{ml}$ de medio de cultivo enmendado. Adicionalmente, se inocularon diez placas de Petri conteniendo los medios sin enmendar (testigos).

La incubación se realizó a $25^{\circ} \mathrm{C} \pm 2^{\circ} \mathrm{C}$ durante 24 días para M. roreri, 12 días para $\mathrm{P}$. palmivora, tiempos en los que el micelio en los testigos cubrieron totalmente la superficie de la placa de Petri (9 $\mathrm{cm}$ de diámetro). Con los datos obtenidos se calcularon los porcentajes de inhibición a través de la fórmula:

Porcentaje de inhibición $=\frac{\text { Diámetro del testigo }- \text { Diámetro del tratamiento }}{\text { Diámetro del testigo }} \times 100$

En aquellos tratamientos/repeticiones en donde no se presentó crecimiento de los microrganismos, se tomaron los discos de micelio usados como inóculo y se colocaron en placas de Petri conteniendo medios estériles, con la finalidad de determinar su viabilidad. Se establecieron los testigos siguiendo el mismo procedimiento del anterior (cultivo del patógeno en medio PDA sin enmendar). La evaluación se realizó una vez que los testigos cubran completamente el medio de cultivo y consistió en registrar el crecimiento (viabilidad) o muerte de los patógenos en cada una de las placas. Aquellas placas en donde no se registró crecimiento se consideró como muerte del micelio y en ese caso se consideró como efecto fungicida. Con los datos obtenidos (número de placas sin crecimiento) se calculó el porcentaje de acción fungicida por cada uno de los extractos y concentraciones usadas:

Porcentaje de acción fungicida $=$

Número de placas con muerte del micelio

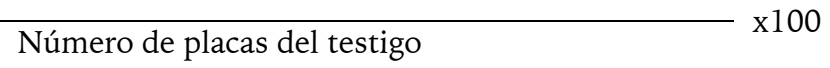

Efecto de extractos vegetales sobre la estructura del micelio de los patógenos

Para evaluar el efecto de los extractos vegetales sobre la estructura del micelio de los patógenos, estos se inocularon en medio de cultivo líquido Papa Dextrosa enmendado con los extractos vegetales en tres concentraciones diferentes: 10, 20 y $30 \% \mathrm{v} / \mathrm{v}$.

Inicialmente, se obtuvieron cultivos del patógeno en PDA y AV8 (P. palmivora) de 21 días de edad. Para ello, a partir del cultivo madre se realizaron repiques a placas de Petri conteniendo $20 \mathrm{ml}$ de medio estéril. Las placas se incubaron a $25^{\circ} \mathrm{C} \pm 2{ }^{\circ} \mathrm{C}$ durante 21 días en oscuridad.

Los medios enmendados se prepararon utilizando medio líquido Papa Dextrosa 100\% al que se le añadió los extractos en las concentraciones antes mencionadas. Se inocularon tres matraces Erlenmeyer de $25 \mathrm{~mL}$ (10 ml de medio líquido cada uno) por cada uno de los extractos y tres concentraciones diferentes, en cada matraz se colocaron dos discos de micelio del patógeno de $5 \mathrm{~mm}$ de diámetro. Adicionalmente, se inocularon tres matraces con medio sin enmendar (testigo). La incubación se realizó a $25^{\circ} \mathrm{C} \pm 2{ }^{\circ} \mathrm{C}$ durante 15 días, al final de los cuales se realizó la evaluación de la estructura del micelio y desarrollo del mismo en los medios líquidos.

Por cada matraz se retiraron los discos de micelio y en los tratamientos con crecimiento micelial se prepararon placas para observación microscópica con aumento de 100X. De 
acuerdo a la estructura del micelio del

los valores de acuerdo a la Tabla 1.

patógeno en cada tratamiento se le asignaron

Tabla 1. Valores utilizados para evaluar la estructura del micelio de $M$. roreri

\begin{tabular}{cc}
\hline Valores & Descripción \\
\hline $0(-)$ & Sin crecimiento de micelio \\
$1(+)$ & $\begin{array}{c}\text { Crecimiento del micelio con estructuras celulares y citoplasma } \\
\text { alteradas, desorganización de los contenidos celulares }\end{array}$ \\
$2(++)$ & Crecimiento de micelio sin alteraciones en el citoplasma \\
\hline
\end{tabular}

Diseño experimental y análisis estadístico

Para el análisis de los datos en cada patógeno, se aplicó un diseño completamente al azar (DCA) con un arreglo factorial $3 \times 6+1$ en 10 repeticiones. Se consideraron dos factores en estudio: concentraciones y extractos, cuyas combinaciones se compararon con un tratamiento control. Para establecer diferencias entre las medias de los tratamientos se hizo uso de la prueba de rango múltiple de Tukey $(\mathrm{P} \leq 0.05)$. El procesamiento estadístico se lo realizó con el software R.

Tratamientos estudiados

La combinación de los dos factores (seis extractos por tres concentraciones) en estudio reflejó dieciocho tratamientos, tal como se describen en la Tabla 2.

Tabla 2. Descripción de los tratamientos evaluados

\begin{tabular}{|c|c|c|c|c|}
\hline Trat & Cod. & Concentración & Extractos & UE \\
\hline T1 & C1E1 & $10 \% \mathrm{v} / \mathrm{v}$ & Bledo (A. bledo) & 10 \\
\hline $\mathrm{T} 2$ & C1E2 & & Coquito (C. odoratus) & 10 \\
\hline T3 & C1E3 & & Lechosa (E. hirta) & 10 \\
\hline $\mathrm{T} 4$ & C1E4 & & Rosa de muerto (T. minuta) & 10 \\
\hline T5 & C1E5 & & Teatina (S. dulcis) & 10 \\
\hline T6 & C1E6 & & Verdolaga (P. oleracea) & 10 \\
\hline $\mathrm{T} 7$ & C2E1 & $20 \% \mathrm{v} / \mathrm{v}$ & Bledo (A. bledo) & 10 \\
\hline T8 & C2E2 & & Coquito (C. odoratus) & 10 \\
\hline T9 & C2E3 & & Lechosa (E. hirta) & 10 \\
\hline T10 & $\mathrm{C} 2 \mathrm{E} 4$ & & Rosa de muerto (T. minuta) & 10 \\
\hline $\mathrm{T} 11$ & C2E5 & & Teatina (S. dulcis) & 10 \\
\hline $\mathrm{T} 12$ & C2E6 & & Verdolaga (P. oleracea) & 10 \\
\hline T13 & C3E1 & $30 \% \mathrm{v} / \mathrm{v}$ & Bledo (A. bledo) & 10 \\
\hline T14 & C3E2 & & Coquito (C. odoratus) & 10 \\
\hline
\end{tabular}




$\begin{array}{llll}\text { T15 } & \text { C3E3 } & \text { Lechosa }(E . \text { hirta }) & 10 \\ \text { T16 } & \text { C3E4 } & \text { Rosa de muerto }(T . \text { minuta }) & 10 \\ \text { T17 } & \text { C3E5 } & \text { Teatina }(S . \text { dulcis }) & 10 \\ \text { T18 } & \text { C3E6 } & \text { Verdolaga }(P . \text { oleracea }) & 10 \\ \text { T19 } & \text { Control } & \text { Testigo sin aplicación de extractos } & 10\end{array}$

\section{RESULTADOS}

En las Tablas 3 y 4 , se presentan los resultados obtenidos al evaluar el efecto de los extractos vegetales sobre la germinación de las esporas de los dos patógenos en estudio. Todos los extractos evaluados tuvieron un efecto sobre los porcentajes de germinación de esporas, observándose que en todos los casos al aumentar las concentraciones disminuye el porcentaje de germinación de las esporas de los patógenos.
Se observó que los extractos que tuvieron mayor efecto sobre la germinación de esporas fueron los de las especies A. bledo, E. hirta y T. minuta. Se destaca que las zoosporas de P. palmivora fueron las más sensibles al efecto de los extractos, dado que la germinación de ellas fue inhibida con la mayor parte de los extractos aun en la menor concentración (10\%). La germinación de esporas en las concentraciones de 20 y $30 \%$ se redujo drásticamente en los todos los tratamientos.

Tabla 3. Efecto de los extractos vegetales sobre la germinación de esporas del patógeno M. roreri

\begin{tabular}{lccccccc}
\hline \multirow{2}{*}{ Especie } & \multicolumn{5}{c}{ Porcentajes de germinación de M. roreri } \\
\cline { 2 - 8 } & \multicolumn{2}{c}{ Medio al $10 \%$} & Medio al 20 \% & Medio al $30 \%$ \\
\hline Bledo (A. bledo) & 0.00 & $\mathrm{~d}$ & 0.00 & $\mathrm{~b}$ & 0.00 & $\mathrm{~b}$ \\
Coquito (C. odoratus) & 21.40 & $\mathrm{c}$ & 0.00 & $\mathrm{~b}$ & 0.00 & $\mathrm{~b}$ \\
Lechosa (E. hirta) & 0.40 & $\mathrm{~d}$ & 0.00 & $\mathrm{~b}$ & 0.00 & $\mathrm{~b}$ \\
Rosa de muerto (T. minuta) & 1.40 & $\mathrm{~d}$ & 0.00 & $\mathrm{~b}$ & 0.00 & $\mathrm{~b}$ \\
Teatina (S. dulcis) & 38.80 & $\mathrm{a}$ & & 6.50 & $\mathrm{a}$ & 1.80 & $\mathrm{a}$ \\
Verdolaga (P. oleracea) & 26.60 & $\mathrm{~b}$ & 0.00 & $\mathrm{~b}$ & 0.00 & $\mathrm{~b}$ \\
\hline
\end{tabular}

Promedios con la misma letra en cada grupo de datos, no difieren estadísticamente según la prueba de Tukey al $5 \%$.

Tabla 4. Efecto de los extractos vegetales sobre la germinación de esporas del patógeno P. palmivora

\begin{tabular}{lrrrrr}
\multirow{2}{*}{ Especie } & \multicolumn{5}{c}{ Porcentajes de germinación de P. palmivora } \\
\cline { 2 - 6 } & \multicolumn{2}{c}{ Medio al 10\% } & Medio al 20\% & Medio al 30\% \\
\hline Bledo (A. bledo) & 0.00 & $\mathrm{c}$ & 0.00 & $\mathrm{~b}$ & 0.00 \\
Coquito (C. odoratus) & 0.00 & $\mathrm{c}$ & 0.00 & $\mathrm{~b}$ & 0.00 \\
Lechosa (E. hirta) & 0.00 & $\mathrm{c}$ & 0.00 & $\mathrm{~b}$ & 0.00 \\
Rosa de muerto (T. minuta) & 0.00 & $\mathrm{c}$ & 0.00 & $\mathrm{~b}$ & 0.00 \\
Teatina (S. dulcis) & 90.60 & $\mathrm{~b}$ & 16.60 & $\mathrm{a}$ & 0.00 \\
Verdolaga (P. oleracea) & 100.00 & $\mathrm{a}$ & 0.00 & $\mathrm{~b}$ & 0.00 \\
\hline
\end{tabular}

Promedios con la misma letra en cada grupo de datos, no difieren estadísticamente según la prueba de Tukey al 5\%. 
En las Tablas 5 y 6 , se muestran los resultados del análisis del efecto de las concentraciones de los extractos sobre la germinación de esporas de los patógenos. En el caso de $\mathrm{M}$. roreri (Tabla 5), los resultados indican que el extracto de A. bledo, E. hirta y T. minuta, no mostraron diferencias significativas entre las concentraciones evaluadas por lo que afectan significativamente la germinación de esporas del patógeno. Para P. palmivora el efecto sobre las esporas se logró con todos los extractos, siendo la especie con menor capacidad inhibitoria sobre la germinación P. oleracea (Tabla 6).

Tabla 5. Efecto de las concentraciones de los extractos vegetales sobre la germinación de esporas in vitro del patógeno M. roreri

\begin{tabular}{|c|c|c|c|c|c|c|}
\hline Concentraciones & A. bledo & C. odoratus & E. hirta & T. minuta & S. dulcis & P. oleracea \\
\hline $\mathrm{C}_{1}: 10 \% \mathrm{v} / \mathrm{v}$ & 0 & $21.4 \mathrm{a}$ & 0.4 & 1.4 & $38.8 \mathrm{a}$ & 26.6 a \\
\hline $\mathrm{C}_{2}: 20 \% \mathrm{v} / \mathrm{v}$ & 0 & 0 & 0 & 0 & 6.5 & $\mathrm{~b}$ \\
\hline $\mathrm{C}_{3}: 30 \% \mathrm{v} / \mathrm{v}$ & 0 & 0 & 0 & 0 & 1.8 & $\mathrm{~b}$ \\
\hline
\end{tabular}

Promedios con la misma letra en cada grupo de datos, no difieren estadísticamente según la prueba de Tukey al 5\%.

Tabla 6. Efecto de las concentraciones de los extractos vegetales sobre la germinación de esporas in vitro del patógeno P.

\begin{tabular}{lcccccccc}
\hline Concentraciones & A. bledo & C. odoratus & E. hirta & T. minuta & S. dulcis & P. oleracea \\
\hline $\mathrm{C}_{1}: 10 \% \mathrm{v} / \mathrm{v}$ & 0 & 0 & 0 & 0 & 90.6 & $\mathrm{a}$ & 100.0 & $\mathrm{a}$ \\
$\mathrm{C}_{2}: 20 \% \mathrm{v} / \mathrm{v}$ & 0 & 0 & 0 & 0 & 16.6 & $\mathrm{~b}$ & 0 & $\mathrm{~b}$ \\
$\mathrm{C}_{3}: 30 \% \mathrm{v} / \mathrm{v}$ & 0 & 0 & 0 & 0 & 0.0 & $\mathrm{c}$ & 0 & $\mathrm{~b}$ \\
\hline
\end{tabular}

Promedios con la misma letra en cada grupo de datos, no difieren estadísticamente según la prueba de Tukey al $5 \%$.

El efecto de los extractos vegetales sobre la inhibición del crecimiento micelial in vitro de los patógenos se presentan en las Tablas 7 y 8 . El crecimiento del patógeno $M$. roreri fue inhibido en todos los extractos y en todas las concentraciones (Tabla 7), siendo la más inhitoria A. bledo y la especie con menor efecto inhibitorio S. dulcis. Cuando se evaluó el crecimiento de P. palmivora, se encontró que nuevamente las especies A. bledo y E. hirta inhibieron al $100 \%$ el desarrollo micelial en las tres concentraciones usadas (Tabla 8).

Tabla 7. Efecto de los extractos vegetales sobre la inhibición del crecimiento micelial in vitro del patógeno M. roreri

\begin{tabular}{|c|c|c|c|}
\hline \multirow{2}{*}{ Extractos } & \multicolumn{3}{|c|}{ Inhibición del crecimiento micelial } \\
\hline & Medio al $10 \%$ & Medio al $20 \%$ & Medio al $30 \%$ \\
\hline Bledo (A. bledo) & $100.00 \mathrm{a}$ & $100.00 \mathrm{a}$ & $100.00 \mathrm{a}$ \\
\hline Coquito (C. odoratus) & 21.29 & 60.00 & 98.24 a \\
\hline Lechosa (E. hirta) & 46.11 & $100.00 \mathrm{a}$ & $100.00 \mathrm{a}$ \\
\hline Rosa de muerto ( $T$. minuta) & 0.00 & 97.64 a & $100.00 \mathrm{a}$ \\
\hline Teatina (S. dulcis) & 0.00 & 12.24 & $100.00 \mathrm{a}$ \\
\hline Verdolaga (P. oleracea) & 0.00 & 60.23 & $100.00 \mathrm{a}$ \\
\hline
\end{tabular}

Promedios con la misma letra en cada grupo de datos, no difieren estadísticamente según la prueba de Tukey al 5\%.

Al evaluar la viabilidad del micelio en las cajas de Petri en donde se registró crecimiento 
en la prueba anterior, se encontró muerte del micelio de P. palmivora en todas las unidades evaluadas lo que indicaría que todos los extractos tuvieron un efecto actifúngico sobre los patógenos. En el caso de M. roreri, se observó un panorama diferente (Figura 1). La actividad antifúngica del extracto de A. bledo fue del $100 \%$ en todas las concentraciones evaluadas, seguida de E. hirta quien presentó efecto antifúngico al 20 y $30 \%$. Las especies C. odoratus, S. dulcis y $\mathrm{P}$. oleracea presentaron crecimiento de micelio, en las concentraciones 10 y $20 \%$ lo que indica que aunque los extractos detienen el crecimiento de los patógenos el micelio permanece vivo.

Tabla 8. Efecto de los extractos vegetales sobre la inhibición del crecimiento micelial in vitro del patógeno P. palmivora

\begin{tabular}{|c|c|c|c|c|c|}
\hline \multirow{2}{*}{ Extractos } & \multicolumn{5}{|c|}{ Inhibición del crecimiento micelial } \\
\hline & $\begin{array}{r}\text { Medio } \\
\% \\
\end{array}$ & 110 & $\begin{array}{r}\text { Medio a } \\
\% \\
\end{array}$ & 120 & $\begin{array}{c}\text { Medio al } 30 \\
\%\end{array}$ \\
\hline Bledo ( $A$. bledo) & 100.00 & a & 100.00 & a & 100.00 \\
\hline Coquito (C. odoratus) & 68.29 & $\mathrm{~b}$ & 88.35 & $b$ & 100.00 \\
\hline Lechosa ( $E$. hirta) & 100.00 & $\mathrm{a}$ & 100.00 & $\mathrm{a}$ & 100.00 \\
\hline Rosa de muerto ( $T$. minuta) & 55.06 & c & 78.34 & $\mathrm{~b}$ & 100.00 \\
\hline Teatina ( $S$. dulcis) & 58.35 & c & 81.00 & $\mathrm{~b}$ & 100.00 \\
\hline Verdolaga ( $P$. oleracea) & 59.41 & C & 73.47 & $\mathrm{~b}$ & 100.00 \\
\hline
\end{tabular}

Promedios con la misma letra en cada grupo de datos, no difieren estadísticamente según la prueba de Tukey al 5\%.

En la Tabla 9, se presentan los cambios generados por los extractos, sobre la estructura del micelio de los patógenos. Los extractos de bledo y lechosa no permitieron el desarrollo de micelio de los patógenos en los medios líquidos enmendados. Mientras que C. odoratus (coquito) cuando fue utilizado para enmendar los medios de cultivo en una concentración al $10 \%$ permitió el desarrollo

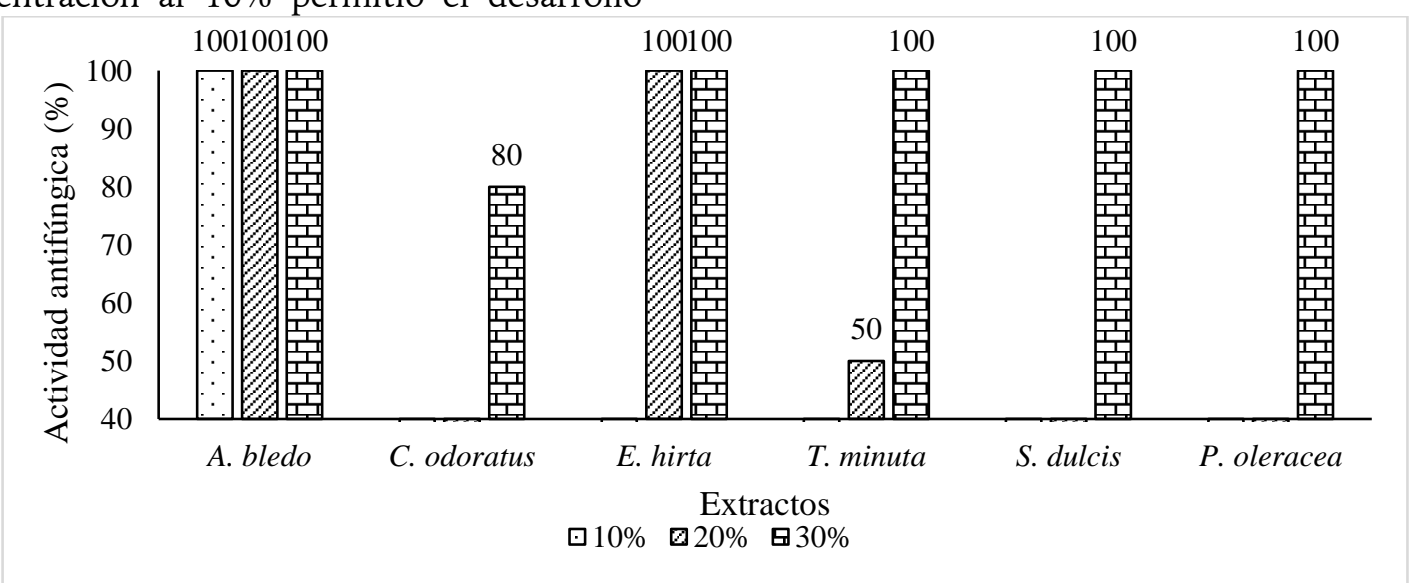

Figura 1. Acción antifúngica (\%) de los extractos vegetales frente al patógeno M. roreri miceliar de todos los patógenos, sin embargo, este micelio presentó alteraciones en sus estructuras internas y desorganización de los contenidos celulares. M. roreri presentó crecimiento sin mostrar alteraciones cuando creció en los medios enmendados con $\mathrm{T}$. minuta y S. dulcis al 10\%,

Tabla 9. Efecto de los extractos vegetales sobre la estructura del micelio de los patógenos. 


\begin{tabular}{|c|c|c|c|}
\hline & Tratamientos & M. roreri & P. palmivora \\
\hline & Control & ++ & ++ \\
\hline & Bledo $10 \%$ & - & - \\
\hline & Bledo $20 \%$ & - & - \\
\hline & Bledo $30 \%$ & - & - \\
\hline & Coquito $10 \%$ & + & + \\
\hline & Coquito $20 \%$ & - & - \\
\hline & Coquito $30 \%$ & - & - \\
\hline & Lechosa $10 \%$ & - & - \\
\hline & Lechosa $20 \%$ & - & - \\
\hline & Lechosa $30 \%$ & - & - \\
\hline & Rosa $10 \%$ & ++ & - \\
\hline & Rosa $20 \%$ & - & - \\
\hline & Rosa $30 \%$ & - & - \\
\hline & Teatina $10 \%$ & ++ & + \\
\hline & Teatina $20 \%$ & - & - \\
\hline & Teatina $30 \%$ & - & - \\
\hline & Verdolaga $10 \%$ & + & + \\
\hline & Verdolaga $20 \%$ & - & - \\
\hline & Verdolaga $30 \%$ & - & - \\
\hline $\begin{array}{l}\text { Sin crecimiento de micelio } \\
\text { celulares ++: Crecimient }\end{array}$ & $\begin{array}{l}+: \text { Crecimiento del micelio con estruct } \\
\text { o de micelio sin alteraciones en el citop }\end{array}$ & elulares y citc & lteradas, desorganización de los contenidos \\
\hline $\begin{array}{l}\text { 3. DISCUSI } \\
\text { Luego de revi } \\
\text { seleccionaron sei } \\
\text { región que son } \\
\text { (Amaranthus blec } \\
\text { coquito (Cyperus } \\
\text { (Tagetes minuta) } \\
\text { verdolaga (Portu } \\
\text { especies seleccior } \\
\text { son utilizadas } \\
\text { medicinales. }\end{array}$ & $\begin{array}{l}\text { ÓN } \\
\text { sar fuentes bibliográficas se } \\
\text { s especies de plantas de la } \\
\text { consideradas malezas: bledo } \\
\text { to), teatina (Scoparia dulcis), } \\
\text { odoratus), rosa de muerto } \\
\text { lechosa (Euphorbia hirta) y } \\
\text { laca oleracea). Dos de las } \\
\text { ladas, S. dulcis y T. minuta, } \\
\text { también como plantas }\end{array}$ & $\begin{array}{l}\text { quino } \\
\text { tanino } \\
\text { grupo } \\
\text { antim } \\
\text { defen } \\
\text { patóg } \\
\text { comp } \\
\text { patóg } \\
\text { en cl } \\
\text { contro } \\
\text { produ } \\
\text { produ } \\
\text { Lo } \\
\text { E. hir } \\
\text { el des } \\
\text { germi } \\
\text { viabili } \\
\text { simila }\end{array}$ & $\begin{array}{l}\text { avonas, flavonoides, flavonoles, } \\
\text { narina (Singh et al., 2012). Estos } \\
\text { ompuestos muestran un efecto } \\
\text { no y sirven como mecanismos de } \\
\text { s plantas contra microorganismos } \\
\text { Algunas plantas contienen } \\
\text { que son tóxicos para los } \\
\text { le cuando se extraen y se aplican } \\
\text { infestados pueden realizar un } \\
\text { ivo de las enfermedades, a estos } \\
\text { los llaman (pesticidas botánicos o } \\
\text { tánicos) (Kumar \& Singh, 2012). } \\
\text { tados demuestran que A. bledo y } \\
\text { el mayor potencial para afectar } \\
\text { de los patógenos, tanto en su } \\
\text { como en el crecimiento y } \\
\text { su micelio. El efecto observado es } \\
\text { e fue reportado por Yusnawan ( }\end{array}$ \\
\hline
\end{tabular}


2015) al evaluar los extractos de una de las especies de Amaranthus (A. espinosus), los mismos que fueron efectivos para disminuir la germinación de esporas de $\mathrm{P}$. pachyrhizi, provocando mayor porcentaje de lisis de esporas del mencionado hongo. El mencionado autor sostiene que Amaranthus sp. contiene alcaloides, flavonoides, taninos, saponinas y terpenoides por lo que puede ser utilizado como fungicida botánico para controlar la germinación de esporas. Esto coincide con lo señalado por Singh \& Kumar (2012), quienes sostienen que compuestos como fenoles, ácidos fenólicos, quinonas, flavonoles, flavonoides, taninos y cumarinas tienen registros de actividad antifúngica, compuestos que forman parte de las plantas y que sirven como mecanismos de defensa frente a los patógenos.

Por otro lado, al evaluarse el crecimiento micelial de los hongos, se observó una tendencia similar que en la germinación, siendo el incremento de la concentración un factor importante para causar un mayor impacto en el crecimiento micelial, de tal manera que al aumentarla, también se incrementa la efectividad en la inhibición del crecimiento micelial. Estos resultados son corroborados por los reportes de Mukherjee et al. (2011), que al incrementar la concentración de los extractos de S. macrophylla, Z. officinale, N. tabacum, C gigantea, A. sativum y S. apetala, desde el 30 al $70 \%$, se incrementó significativamente la inhibición del crecimiento micelial de Colletotrichum gloeosporioides desde el $21.51 \%$ hasta un $41.04 \%$.

En investigaciones similares, extractos de especies de malezas como Cyperus rotundus inhibieron la germinación de esporas y/o desarrollo de micelio de Colletotrichum gloesporoides y Curvularia spp (Meera \& Srivastava, 2019), Fusarium udum (Singh et al., 1999), Puccinia arachidis ( Yusnawan \& Inayati, 2018) entre otros patógenos; resultados similares han sido observados por extractos obtenidos a partir de Amaranthus spp. frente a patógenos como Alternaria alternata, Fusarium solani, Candida albicans, Fusarium oxysporum, Trichoderma sp. y Aspergillus ochraceus (Rivillas \& Soriano, 2007); Tagetes minuta frente a Rhizoctonia solani, Sclerotinia sclerotiorum y Sclerotium rolfsii (Saha et al., 2012); Euphorbia hirta frente a Fusarium moniliforme y Phoma sorghina (Karanga et al., 2017).

Finalmente, los daños ocasionados tanto por el extracto de A. bledo, así como el de E. hirta desde la concentración más baja hasta la más alta en estudio, fueron eficientes al ocasionar alteraciones en dicha estructura micelial, produciendo la inhibición total del crecimiento del micelio. Esto cobra importancia, y a la vez exige verificar los cambios de los extractos sobre la integridad de la membrana plasmática, puesto que ésta tiene un papel crucial en el mantenimiento de la viabilidad fúngica (Shao et al., 2013; Tao et al., 2014). Existen indicios que demuestran que los aceites esenciales de plantas pueden aumentar la permeabilidad de la membrana celular y dañar la integridad de la membrana al disminuir los componentes importantes de la estructura de la membrana celular, los lípidos totales y el ergosterol, tal como fue indicado por Shreaz et al. (2016).

\section{CONCLUSIONES}

La literatura científica indica que varias de las especies de plantas algunas de ellas consideradas malezas en nuestra región tienen potencial para el control de enfermedades de plantas debido a la presencia de compuestos antifúngicos, tales como: A. bledo, E. hirta, C. odoratus, S. dulcis, T. minuta y P. oleracea. Todos los extractos evaluados tuvieron efecto sobre la germinación de esporas de los cuatro patógenos evaluados, destacándose el efecto de A. bledo y E. hirta sobre las demás especies. Al igual que en el caso de la germinación, los extractos de las especies evaluadas ejercieron un efecto negativo sobre el desarrollo y viabilidad del micelio de los patógenos, llegando inclusive tener un impacto en la estructura del micelio mostrando actividad 
antifúngica, especialmente en el caso de las especies A. bledo y E. hirta. Los resultados obtenidos a nivel in vitro muestran una guía en la búsqueda de soluciones para reducir el impacto de los patógenos que inciden directamente en las mazorcas de cacao, experimentos in vivo son necesarios para evaluar su comportamiento y desempeño a nivel de campo.

\section{AGRADECIMIENTOS}

La información presentada forma parte de los resultados obtenidos en el proyecto de investigación APROVECHAMIENTO DE LA ACTIVIDAD ANTIFÚNGICA DE PLANTAS PARA EL CONTROL DE ENFERMEDADES EN CULTIVOS DE INTERÉS ECONÓMICO financiado por la Universidad Técnica Estatal de Quevedo a través de su Fondo Competitivo de Investigación Científica y Tecnológica "FOCICYT". Los autores expresan su agradecimiento al Doctor Eduardo Díaz y Doctor Byron Oviedo por la promoción y gestión de recursos destinados para la investigación en la institución.

\section{REFERENCIAS}

[1]. Balandrin, M., Klocke, J., Wurtele, E., \& Bollinger, H. 1985. Natural Plant Chemicals: Sources of Industrial and Medicinal Materials. Sciencie, 228, 1154-1160.

[2]. Isman, M. 2006. Botanical insecticides, deterrents, and repelents in modern agriculture and an increasingly regulated world. Annu. Rev. Entomol., $51,45-66$.

[3]. Kumar, P. (2013). Antifungal activity of some common weed extracts against wilt causing fungi, Fusarium oxysporum. ResearchGate, 2 (1)(6267).

[4]. Meera, P., \& Srivastava, J. (2019). Antifungal activity of the ethyl acetate extract of Cyperus rotundus rhizome against certain fungi responsible for food spoilage and plant diseases. International Journal of Food Science and Nutrition, 4(3): 88-89.

[5]. Mukherjee, A., Khandker, S., Islam, M., \& Shahid, S. (2011). Efficacy of some plant extracts on the mycelial growth of Colletotrichum gloeosporioides. Journal of the Bangladesh Agricultural University 9(1): 43-47.

[6]. Rivillas, L., \& Soriano, M. (2007). Antifungal Activity of a Protean Extract from Amaranthus hypochondriacus Seeds. Sociedad Química de México, 51 (3) (136.140).

[7].Saha, S., Walia, S., Kundu, A., Kumar, B., \& Joshi, D. (2012). Antifungal acetylinic thiophenes from Tagetes minuta: potential biopesticide. Journal of Applied Botany and Food Quality 85: 207-211.

[8]. Shao, X., Cheng, S., Wang, H., Yu, D., \& Mungai, C. (2013). The possible mechanism of antifungal action of tea tree oil on Botrytis cinerea. Journal of Applied Microbiology 114: 1642-1649.

[9].Shreaz, S., Wani, W., Behbehani, J., Raja, V., Irshad, M., Karched, M., Hun, L. (2016). Cinnamaldehyde and its derivatives, a novel class of antifungal agents. Fitoterapia jul-2016: 116-131.

[10]. Singh, P. K. (2013). Phytochemical study and screening for antimicrobial activity of flavonoids of Euphorbia hirta. International Journal of Applied and Basic Medical Research, 3(2)Singh, M., Ali, S., Akhtar, M., \& Suraj, K. (2012). Efficacy of plant extracts in plant disease management. Agricultural Sciences, 3(3).

[11]. Singh, K. \& Kumar, P. (2013). Ecofriendly Innovative Approaches in Plant Disease Management. International Book Distributors.

[12]. Singh, U., Prithiviraj, B., Khiste, S., Kumar, V., Srivastava, J., \& Manickam, M. (1999). Effect of Cyperus rotundus rhizome extract on Fusarium udum. Indian Phytopath, 52 (1): 18-23. 
[13]. Tao, N., \& Jing, G. (2014). Transcriptional profiling analysis of Penicillium digitatum, the causal agent of citrus green mold, unravels an inhibited ergosterol biosynthesis pathway in response to citral. BMC Genomics 17(1):599.

[14]. Tao, N., OuYang, Q., \& Jia, L. (2014). Citral inhibits mycelial growth of Penicillium italicum by a membrane damage mechanism. Food Control 41: 116-121.

[15]. Ten Hoopen, M. \& Krauss, U. (2016). Biological control of cacao diseases. En B. Bailey, \& L. Meinhardt, Cacao Diseases a History of Old Enemies and New Encounters (pág. 630). New York : Springer Cham Heidelberg.

[16]. Yusnawan, E. (2014). The effectiveness of methanolic and nhexane extracts of Amaranthus spinosus to control Puccinia arachidis and phytochemical screenings of active compounds (In Bahasa Indonesia). En N. Saleh, A. Harsono, N. Nugrahaeni, R. A, J. Sholihin, M. Heriyanto. National Seminar of Legumes and Tuber Crops (págs. 399-405). Malang-Indonesia: ICFCRD.

[17]. Yusnawan, E. (2015). Inhibition of Spore Germination of Phakopsora pachyrhizi Using Crude Extracts of Amaranthus spinosus. Procedia Food Science 3: 340-347.

[18]. Yusnawan, E., \& Inayati, A. (2018). Antifungal Activity of Crude Extracts of Ageratum conyzoides, Cyperus rotundus, and Amaranthus spinosus Against Rust Disease. AGRIVITA Journal of Agricultural Science, 